EPiC Series in Engineering
Volume 3, 2018, Pages 438-446
HIC 2018. 13th International
Conference on Hydroinformatics

\title{
A Customized GIS-based Model for Stormwater Mitigation by LID Controls
}

\author{
Enrico A. Chiaradia ${ }^{1}$, Giulia Ercolani ${ }^{2}$, Gian Battista Bischetti ${ }^{1}$, Claudio \\ Gandolfi $^{1}$, Fabio Castelli ${ }^{2}$, and Daniele Masseroni ${ }^{1}$ \\ 1 Department of Agricultural and Environmental Sciences, University of Milan, Milan, Italy \\ enrico.chiaradia@unimi.it, bischetti@unimi.it, claudio.gandolfi@unimi.it, \\ daniele.masseroni@unimi.it \\ 2 Department of Civil and Environmental Engineering, University of Florence, Florence, Italy \\ giulia.ercolani@dicea.unifi.it, fabio.castelli@unifi.it
}

\begin{abstract}
The effects of urbanization on hydrology, water quality, habitats, as well as ecological and environmental compartments, represent issues of primary importance for multiple agencies at the national, regional and local levels. In the context of the SMART-GREEN project, funded by Fondazione Cariplo, a new tool called SMARTGREEN plugin is under development in a desktop GIS framework. The software will provide: 1) a user friendly interface to help analysts in the hydrologic-hydraulic modelling of urban watersheds and drainage networks through the model MOBIDIC-U, with the possibility of considering Low Impact Development (LID) solutions, 2) a set of tools to easily import information from existing databases, 3) a set of tools to check the database quality, highlight missing or incorrect data, and suggest possible fixes automatically, 4) an easy and faster way to speed up the analysis of the results. In this work, we show the main functionalities of the plugin through a basic test case. The software aims at supporting water service management companies in planning LID implementation in urban areas.
\end{abstract}

\section{Introduction}

The increasing urbanization that many Italian cities are often experiencing causes adverse effects on water quantity and quality, such as augmented runoff volume and rates, decreased runoff lag-time and groundwater recharge, and impaired water quality. In addition, in Italy the areas prone to significant flood risk (combined also with landslide risk) exceed $29500 \mathrm{~km}^{2}$ (about $9.8 \%$ of the Italian territory) and affect more than 6600 municipalities (about 82\%) [8]. Hence, the need to manage rainwater and reduce runoff in the urban context has led many Italian water management companies to increasingly promote directives addressed to the concept of "hydrologic-hydraulic invariance", such as the Lombardy Region law no of 8 April 2016. The "hydrologic-hydraulic invariance" principle requires that the peak flow generated by any area subjected to a land use change has to remain unvaried after the transformation. The adoption of this concept implies to design appropriate mitigation or compensatory measures, and to 
define their allocation at the urban catchment scale in order to decrease the runoff where most needed. Therefore, if on one hand urban Low Impact Development solutions (LIDs), such as bioretention systems, porous pavements,permeable patios, rain barrels/cisterns, green roofs, wet ponds, anddry ponds, appear to be the best practices for reducing stormwater runoff and to improve water quality, on the other hand water manager technicians need an easy to use tool able to design and plan the allocation of these systems in compliance with law recommendations. In order to fulfill this need, the University of Milan and the University of Florence, in collaboration with the water management company of the Milan Metropolitan area (CAP Holding spa) and the Environmental general direction of Lombardy Region, developed the SMART-GREEN project, whose purpose is to implement a software that may support designers and planners in structuring LID actions at urban catchment scale, by investigating the impacts on peak runoff and pollutant attenuations of different requalification scenarios. Despite the project is still in progress, in this work we present the main software components, the flowchart of a simulation work, and the structure of the user interface package at the current level of implementation.

\section{Software architecture}

The SMARTGREEN software has been developed as an extension of the widespread GIS desktop platform QGIS through the plugins technology. QGIS is a well-structured, robust and constantly maintained project that collects an increasing number of endorsements from the worldwide community. Through the SMARTGREEN plugin, we would like to extend the primary functionalities of a GIS platform (data collecting, analysis and reporting) with some custom tools that may support water management companies in the process of building and simulating different land uses scenarios and LIDs planning. In detail, the SMARTGREEN plugin will provide: 1) a user friendly interface to help analysts in performing hydrologic-hydraulic simulations of urban watersheds and drainage networks through the model MOBIDIC-U [4], 2) a set of tools to easily import information from existing databases, 3) a set of tools to check the database quality, highlight missing or incorrect data, suggest possible fixes automatically, 4) an easy and faster way to speed up the analysis of the results. SMARTGREEN plugin is developed in python language and supports, among other GIS file formats, sqlite files to store data.

\subsection{Database structure}

The software describes the complexity of a urban drainage system and its contributing watershed through a set of information contained in a database organized according to the scheme of Figure 1. Links and nodes are the basic geometrical elements representing the drainage system. In the GIS framework, they are connected in term of both geometrical intersections and alphanumeric codes, which are contained in the attributes NODE_START, NODE_END and $O B J_{-} I D$ for links and nodes respectively. The links table contains also the elevation of the element at the starting and ending point (ELEV_START, ELEV_END), the shape of the conduit section (S_SHAPE), the size of the section (DIAM, DIM1, DIM2, etc.), the Manning's roughness coefficient $(M A N N)$ and the length of the conduit $(L E N G T H)$. The nodes are the elements collecting surface runoff and connecting two or more links, and they are characterized by bottom and top elevation ( $\left.E L E V_{-} B O T, E L E V_{-} T O P\right)$ and average storing capacity per unit of depth (AREA). Land characteristics are mainly contained in the SUBCATCHMENTS layer, that defines both the extension of the draining area and its main characteristics through the 


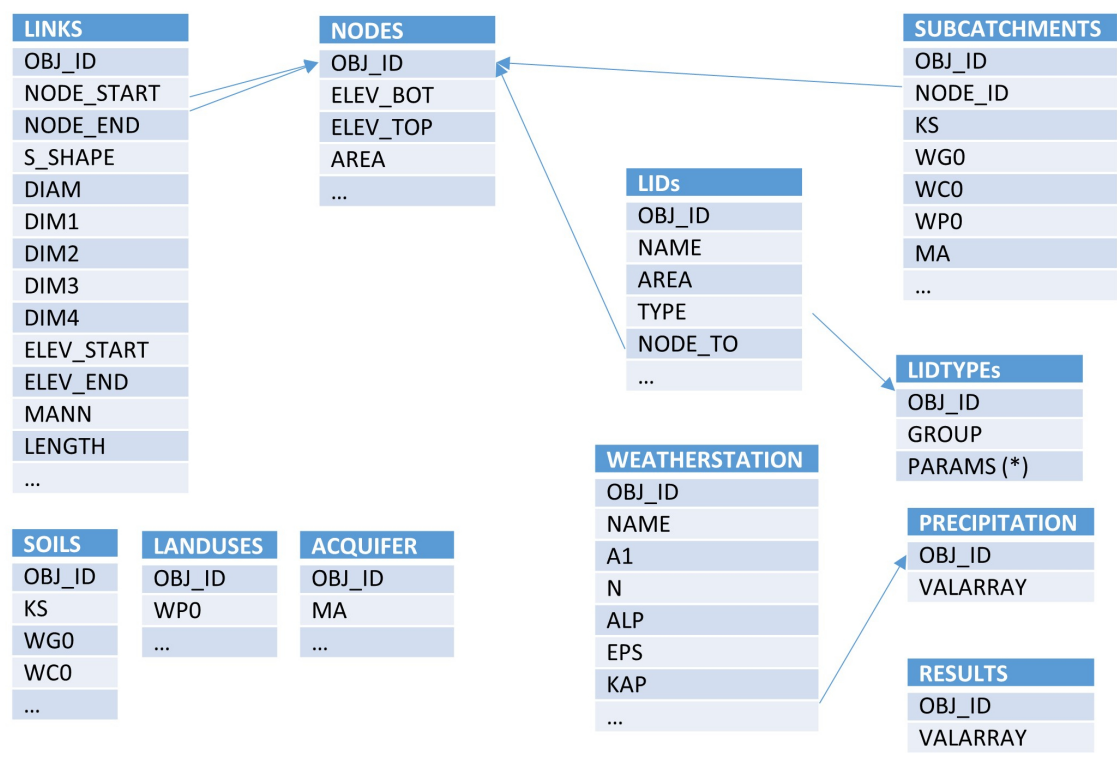

Figure 1: Simplified database structure ( see Figure 2).

attributes $K S, W G 0, W C 0, W P 0$ and $M A$ (soil hydraulic conductivity, soil water holding capacity in large and small pores, rainfall interception capacity, presence of the aquifer). SOILS, LANDUSES and ACQUIFER layers allow to represent local characteristics in terms of hydrological properties. Information about meteorological forcings are stored in the WEATHER $S T A T I O N S$ layer, which, at any available weather station, may contain the parameters of the Intensity-Duration-Frequency (IDF) rainfall curves in accordance with [5] (A1, N, ALP, EPS, $K A P)$. LIDs are represented by polygons, with the possibility of being connected to a specific node (NODE_TO) of the drainage network. LIDs characteristics are defined by a set of parameters (Figure 2). The CATEGORY attribute defines the type of LID (e.g. green roofs, permeable surfaces, dry well, etc.), and the other attributes will be filled according to it. SOIL_IDs lists the type of soils that compose the LID, when appropriate (i.e. in case of green roofs or permeable surfaces). The soil characteristics corresponding to each soil type are archived in a dedicated table, i.e. the "LID soils"table. The same rationale applies to the type of covering vegetation ( VEG_ID attribute and "LID vegetation"table) and the type of sublayer ( $S U_{-} I D$ and "LID sublayer"). In case of dry wells, reservoirs or similar LIDs, the attributes to be defined are: volume and diameter of the LID, diameter of the outlet conduit if present, saturated hydraulic conductivity of the draining material if applicable (VOLMAX, DIAM, DIAM_OUT, DRAINKS respectively). Input and output time series (VALARRAY) are collected in specific tables (i.e. PRECIPITATION and RESULTS).

\subsection{SMARTGREEN overview}

The SMARTGREEN plugin makes available a set of tools useful to create, manage and perform a hydrological simulation through the MOBIDIC-U model. The complete flowchart of a simulation work is shown in Figure 3. The user starts defining a new QGIS project, and, in particular, its name and reference system: the first attribute is necessary to setup the working 


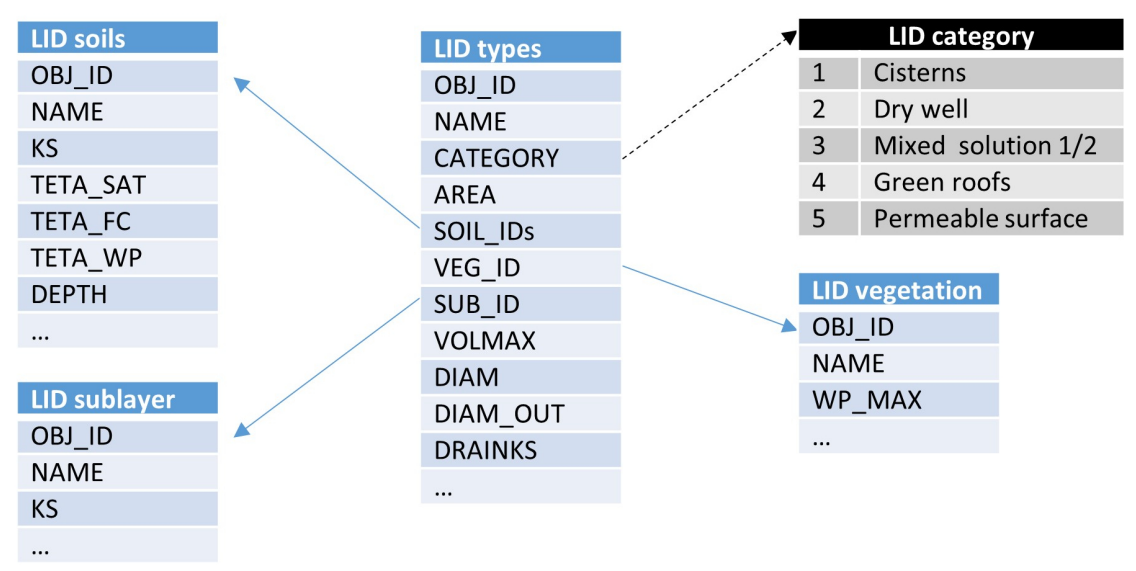

Figure 2: LIDs representation in the SMARTGREEN plugin.

directory where the dataset and the intermediate files will be saved, the second is required in order to ensure the topological coherence of the database. The first elements to be set are nodes and links that describe the urban drainage network. This process can be done manually, using the editing tool provided by QGIS, powered by custom forms that help the user to make the input process faster and safer. Alternatively, the user can import the dataset directly from external sources (vector layers). In this case, first at all, it is suggested to use the "Select upstream"tool in order to highlight all the network draining towards a specific point (the outlet). Consequently, the nodes that will be imported are chosen on the base of the network topology. Thereafter, the corresponding contributing area layer may be created automatically, as well as all the other spatially distributed attributes (land uses, soils and aquifer characteristics). Then, precipitation time series must be included in the project. In particular, at least one meteorological station (i.e. the point to which the time series refers) needs to be defined inside the simulated area. The time series may be imported from an external source, or generated from parameters describing the IDF curves associated to any station. In the latter case, an automatic procedure automatically computes the rainfall input on the basis of the user-defined return period, event duration and type of hyetograph (uniform or Chicago). LIDs are finally represented by polygons corresponding to the areas whose surface runoff is managed through the LID structure (e.g. a parking area whose runoff is conveyed to a water tank). LIDs characteristics are defined in a specific table with general and user defined types. The second main block of the procedure is strictly related to the hydrological analysis. On the basis of the data imported in the project, the SMARTGREEN plugin sets-up automatically all the input files required by the model MOBIDIC-U, and starts the simulation. Finally, simulation results are imported in the GIS framework for a user-friendly visualization: they may be plotted as maps in a spatially distributed view, or as time series selecting a specific position (node or link) in the drainage network.

\subsection{The hydrological model MOBIDIC-U}

The model connected to the SMARTGREEN plugin is MOBIDIC-U (MOdello di Bilancio Idrologico DIstribuito e Continuo - aree Urbane), which simulates runoff formation and propagation in the urban context, including the effect of LID systems [4]. It originates from the distributed 


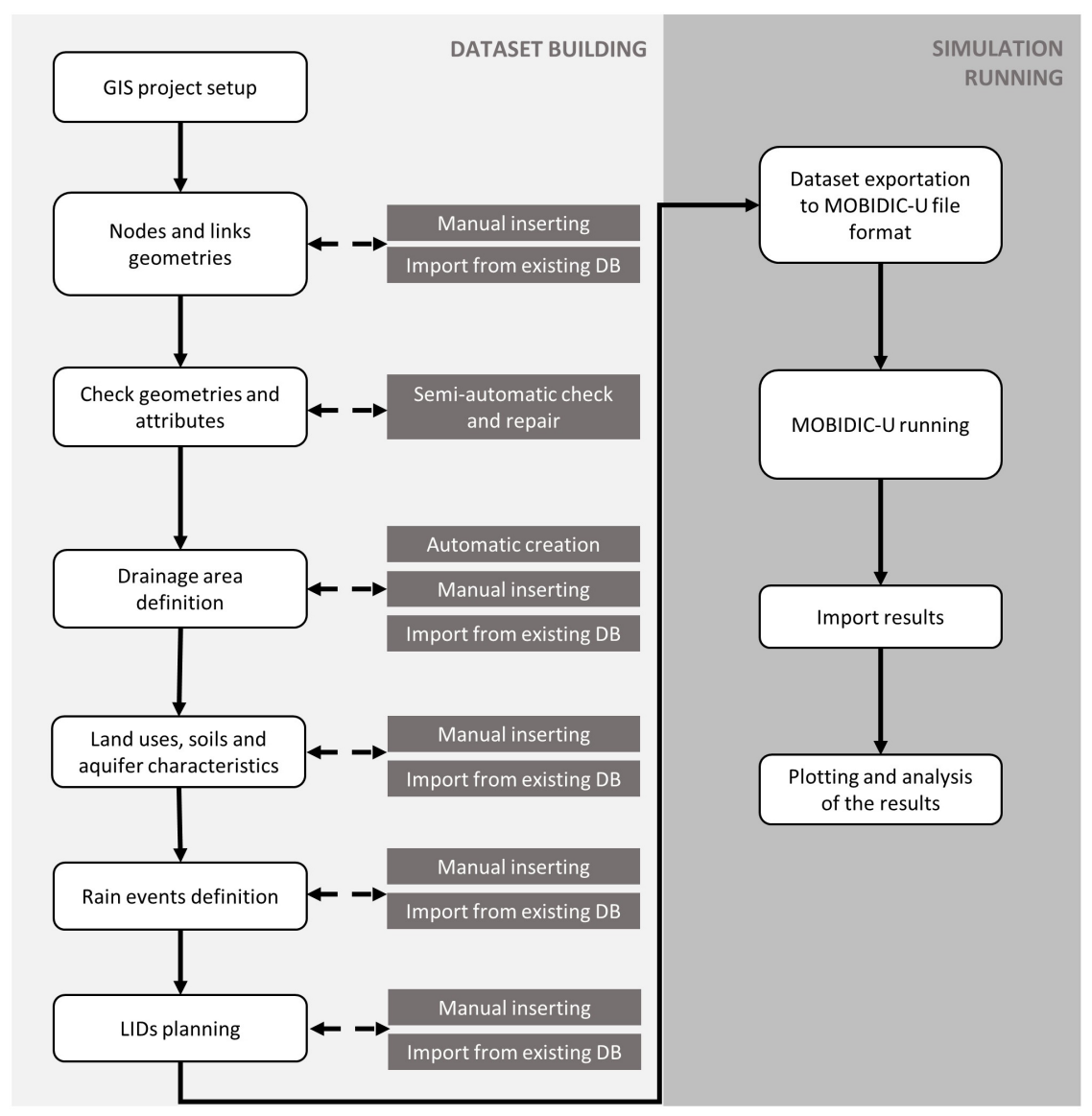

Figure 3: SMARTGREEN plugin workflow and functionalities.

hydrologic model MOBIDIC (MOdello di Bilancio Idrologico DIstribuito e Continuo) [1, 3], which runs operatively at the Hydrologic Service of Tuscany Region (Italy) for flood forecasting and water resources management purposes. MOBIDIC is primarily designed to deal with basins draining towards a natural channel network in accordance with the terrain slope. MOBIDIC-U shares with MOBIDIC the basic modeling approach, but specific modules have been added to adapt the model to the urban context. In particular, the following characteristics are borrowed from MOBIDIC: (i) a fully distributed approach for both parameters and results, with a raster-based horizontal discretization of the area of study, and a vector-based description of the drainage network; (ii) a computationally efficient representation of soil moisture dynamics and surface runoff formation, with each computational cell composed of 5 interconnected conceptual reservoirs (interception reservoir, surface ponding reservoir, gravitational soil reservoir, capillary soil reservoir, phreatic aquifer reservoir). The peculiar characteristic of this approach is the subdivision of the single layer of soil into 2 non-linear reservoirs, the first one corresponding to large pores that drain water under gravity, and the second one corresponding to smaller pores that hold water through capillary forces. A more detailed description of the approach can be found in [2]. The following modeling capabilities are instead specific of MOBIDIC-U, and have been implemented during the SMART-GREEN project: 1) drainage network described 
through a couple of interdependent vector layers, one polyline-type, representing conduits, and one point-type, representing manholes (the nodes of the network). The layers should include both topological data (connection between nodes and branches of the network), and attributes such as manholes top and bottom elevation, conduits slope, material and cross section shape and size; 2) flow routing through the drainage network employing De Saint Venant equations in the conduits and mass conservation in the nodes. The algorithm is capable of dealing with a complex network topology, e.g. including closed circuits; 3) routing of surface runoff and hypodermic flow, from the production cell to the receiving node of the drainage network, taking into account that flow path in the urban environment is barely determined by terrain slope (e.g. presence of elevated surfaces such as roofs); 4) modules simulating the functioning of the main LID systems (e.g. green roofs, permeable pavements, dry wells, water tanks) in terms of hydrologic fluxes and hence surface runoff formation. Although potentially able to run continuous simulations over long periods, MOBIDIC-U is specifically designed for event based runs.

\section{Application test}

In the following we show the main characteristics of the SMARTGREEN plugin through an application test. The plugin is employed to manage a typical assessment of urban development impact on a pre-existent drainage network, and possible mitigation strategies through LID solutions. The case study is a neighbourhood in Sedriano municipality, close to Milan. The area is selected because insufficiencies of the drainage system have been reported, and are probably related to the very fast urbanization experienced in the last years (Figure 4). In addition, the limited extension of both the network and the contributing area, allows to better focus on the functionalities of the plugin. When starting a new job, the plugin populates the QGIS project with a set of layers and tables containing default parameters. Most of the layer are empty, and they will be filled during the analysis process, while others represent the base dataset (Figure 5a). With the "select upstream"tool, it is possible to extract automatically the portion of the network draining towards a specified outlet froma pre-existent couple of lines (the links) and points (the nodes) layers. In our case study, both links and nodes parent layers are provided by the water management company, CAP Holding spa, and are already organized as required by the plugin, i.e. in accordance with Lombardy regional law directives [6]. The attribute table of the links layer may be automatically enriched with details such as roughness coefficient and cross-section shape through the "join tables"tool of the plugin. The joined tables are created by the plugin at the beginning of a new job in conformity with the Lombardy regulation [6], but are easily customizable. In the present test, the "select upstream" tool extracts 26 nodes and 23 links, for a total length of $921.69 \mathrm{~m}$. The shortest elements is $3.94 \mathrm{~m} \mathrm{long}$, while the longest is $98.12 \mathrm{~m}$, and the average length is $40.07 \mathrm{~m}$. The plugin provides a tool to check the consistency of the selected set of nodes and links (Figure 5b). In this case, it returns that the selected network is well structured (i.e. no duplicated or missing geometries exist) but some incomplete or incoherent data are present: 13 links lacks elevation information (7 are elevation at start point), 12 nodes have errors in the bottom elevation values ( 7 with an empty value, the others are higher than the lowest elevation of connected links), 2 nodes lacks the top elevation, all the nodes have no information about the average storing capacity per unit of depth. The plugin includes also a tool to fix automatically the highlighted inconsistencies (Figure 5b), e.g. missing links elevations are recovered from connected nodes, incoherent nodes bottom elevation is fixed on the basis of connected links elevations, nodes storing capacity per unit of depth is set to a default value, etc. After consolidating the network, it is possible to create a first sketch of the drained basin with the "create subcatchments"menu option. In 


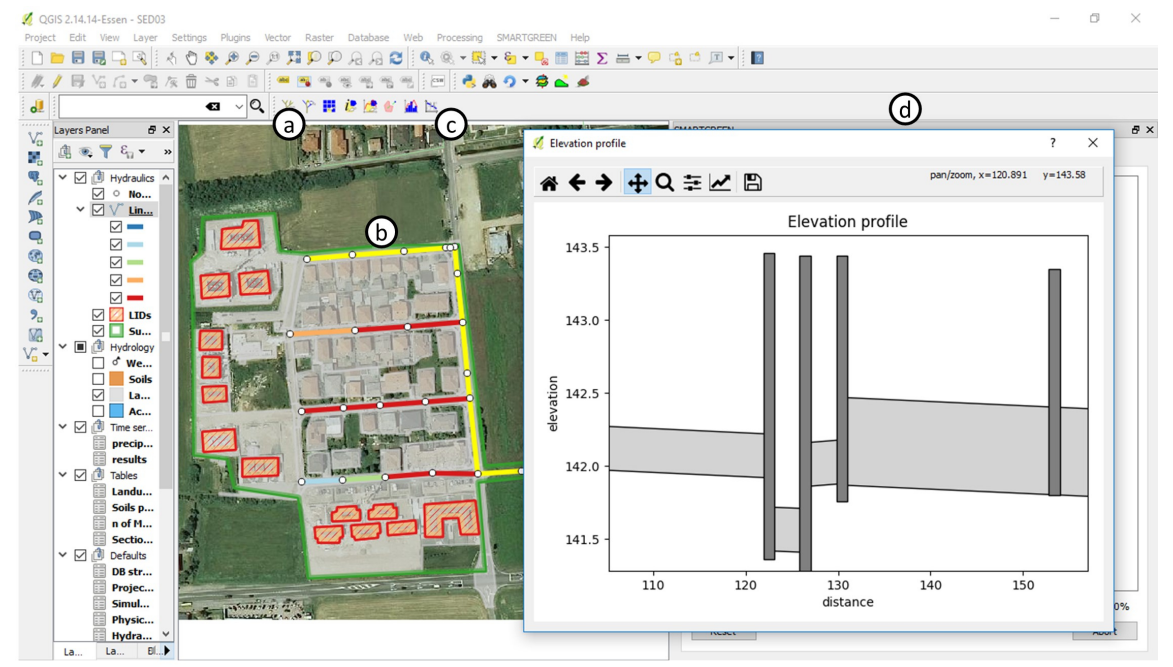

Figure 4: SMARTGREEN plugin tool for network analysis: a) select downstream, b) selected path, c) create elevation profile tool, d) part of the elevation profile with elevation jumps.

this test case, the proposed basin has been modified manually using the default QGIS vector editing tools in order to better fit the actual extension of the urbanized area as shown in a photograph survey dated 2006. The final contributing area has an extension of 3.92 ha. By default, two classes of hydrologic parameter are proposed by the plugin, corresponding to impervious surfaces and a generic pervious land cover [4]. However, any number of classes may be identified by the user, and the proposed parameters are easily modifiable. In the present test, the default classes are maintained, and impervious patches are manually digitized on the basis of the photograph information from 2006. Note that a visual comparison between model setup and ortophotographs is straightforward and immediate at any step thanks to the fact that the software is developed in the QGIS framework. Finally, weather stations are imported from a customized layer of IDF curves deriving from the rasters provided by ARPA Lombardia [5]. Two stations are selected for the study area, and a uniform rain event with a duration of 35 minutes and a return period of 2 years is generated automatically. At this point is possible to export the dataset and run MOBIDIC-U (Figure 5c). Results are then imported back in the QGIS project for a user-friendly visualization. The same procedure is applied to simulate the impact of: 1) urbanized areas expansion during the period 2006-2012;2) impervious roofs transformation in green roofs. Land use transformation areas (case 1) or LIDs surfaces (case 2) are efficiently included in the model setup by simply uploading aerial photographs dated 2012 (www.pcn.minambiente.it) in the GIS project. A default structure for green roofs [7] is already included in the SMARTGREEN plugin. Results can be analysed in different ways. For instance, the plugin can show the maximum degree of filling reached in each link during the simulated rainfall event (Figure 5e), allowing to highlight oversized or undersized conduits at a glance. It is then possible to plot the hydrograph in a specific conduit (Figure 5d), or the water level time series in a node, by simply clicking in the corresponding element. This immediate and intuitive results interrogation should foster the understanding of land use transformation or LIDs introduction at the district scale (Figure 5f). 


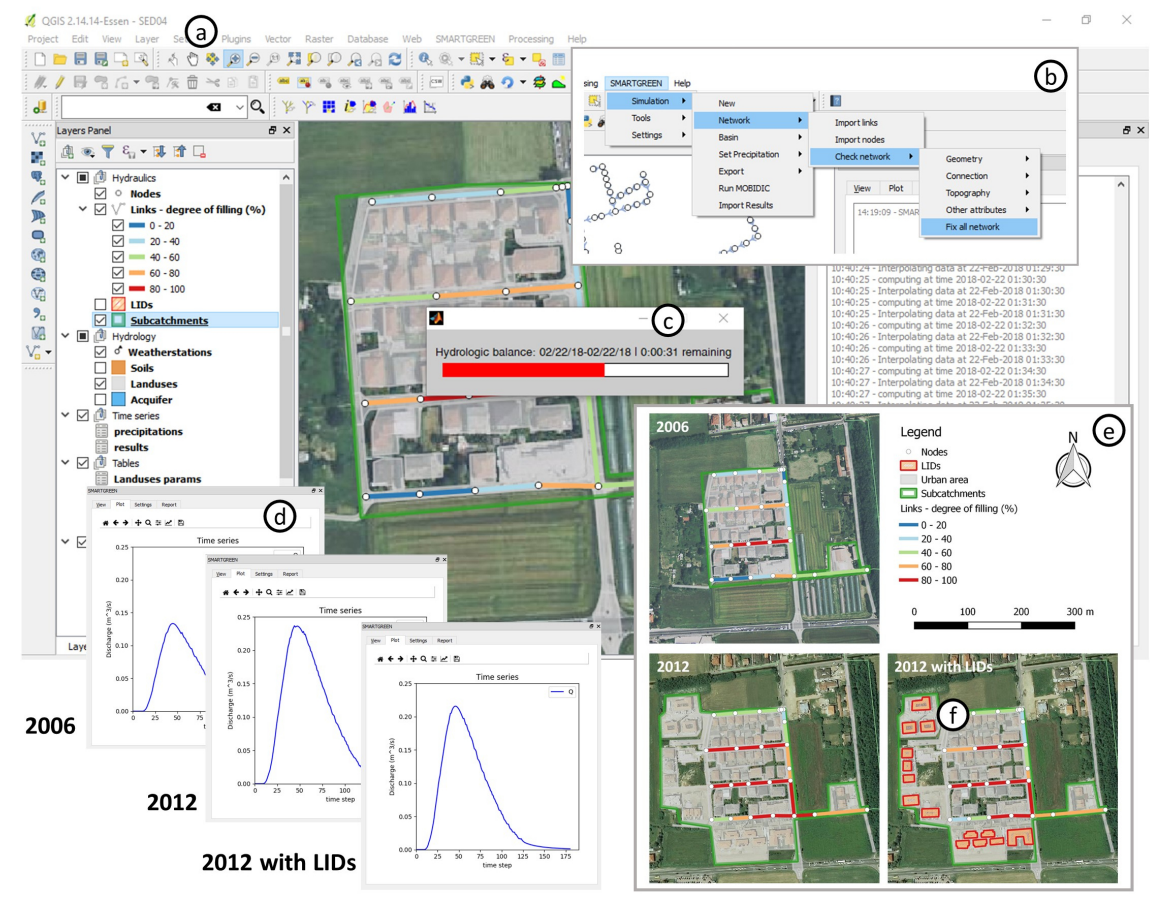

Figure 5: Mosaic of some steps in the use of the SMARTGREEN plugin: a) general GIS framework, b) SMARTGREEN menu items, c) MOBIDIC-U running, d) hydrograph for different scenarios, e) map of conduits degree of filling, f) LIDs planning.

\section{Conclusions}

This paper describes the structure of the SMARTGREEN plugin, a set of tools for the analysis and simulation of urban watersheds and drainage networks fully integrated in a GIS framework. In particular, the plugin aims at offering a user-friendly environment to assess the impact of land uses transformations and LIDs implementation scenarios on urban drainage systems. In this work, through an application to a real case study, it is demonstrated that the provided tools facilitate significantly the setup and run of the distributed hydrologic-hydraulic model included in the plugin (MOBIDIC-U), as well as the visualization of the results. The plugin creates the structure of all the layers needed by the model to describe the urban system, and partially fills them with default values. Then, it can automatically extract the case study from parent layers, detect inconsistencies or missing data in the selected network, fix the highlighted flaws, delineate a first guess contributing area, generate a consolidated dataset as needed by MOBIDIC-U, and show the results through maps and time series. In addition, the advantages related to the full integration of the modelling system in a GIS environment are proved, showing that a proper model setup which reproduces the spatial distribution of historical or novel urbanized areas, as well as of LID solutions compatibly with the current urban pattern, is straightforward thanks to the possibility of uploading georeferenced images in the same environment where the model dataset is generated.

The plugin may hence be considered a valuable support to plan and evaluate LID structures implementation at the urban watershed scale. It is open source and will be freely downloadable 
on a specific web site at the end of the project, whereas currently it can be obtained contacting the authors. Future developments will enrich the software with new functionalities, such as modules for the analysis of costs, the optimization of LIDs spatial distribution, and the simulation of pollutants fate and transport from the modelling point of view.

\section{References}

[1] Fabio Castelli, Giovanni Menduni, and Bernardo Mazzanti. A distributed package for sustainable water management: a case study in the Arno basin. IAHS Publ., 327:52-61, 2009.

[2] Aldrich Castillo, Fabio Castelli, and Dara Entekhabi. Gravitational and capillary soil moisture dynamics for distributed hydrologic models. Hydrology and Earth System Sciences, 19(4):1857, 2015.

[3] Giulia Ercolani and Fabio Castelli. Variational assimilation of streamflow data in distributed flood forecasting. Water Resources Research, 53(1):158-183, 2017.

[4] Giulia Ercolani, Daniela Masseroni, Enrico A. Chiaradia, Gian Battista Bischetti, Claudio Gandolfi, and Fabio Castelli. MOBIDIC-U: a watershed-scale model for stormwater attenuation through green infrastructures design. In AGU Fall Meeting Abstracts, 2017.

[5] ARPA Lombardia. Il monitoraggio degli eventi estremi come strategia di adattamento ai cambiamenti climatici. le piogge intense e le valanghe in Lombardia, 2013.

[6] Regione Lombardia. Modifiche all'allegato 2 del regolamento regionale 15 febbraio 2010, n. 6 recante "Criteri guida per la redazione dei pianiurbani generali dei servizi nel sottosuolo (PUGSS) e criteri per la mappatura e la georeferenziazione delle infrastrutture", 2014.

[7] Anna Palla, Ilaria Gnecco, and Luca Giovanni Lanza. Unsaturated 2D modelling of subsurface water flow in the coarse-grained porous matrix of a green roof. Journal of Hydrology, 379(1-2):193-204, 2009.

[8] Paola Salvati, Cinzia Bianchi, Mauro Rossi, and Fausto Guzzetti. Societal landslide and flood risk in italy. Natural Hazards and Earth System Sciences, 10(3):465-483, 2010. 